PROCEEDINGS OF THE

AMERICAN MATHEMATICAL SOCIETY

Volume 133, Number 10, Pages 3047-3056

S 0002-9939(05)07852-4

Article electronically published on March 31, 2005

\title{
THE BACKWARD SHIFT ON DIRICHLET-TYPE SPACES
}

\author{
STEPHAN RAMON GARCIA
}

(Communicated by Joseph A. Ball)

\begin{abstract}
We study the backward shift operator on Hilbert spaces $\mathcal{H}_{\alpha}$ (for $\alpha \geq 0$ ) which are norm equivalent to the Dirichlet-type spaces $D_{\alpha}$. Although these operators are unitarily equivalent to the adjoints of the forward shift operator on certain weighted Bergman spaces, our approach is direct and completely independent of the standard Cauchy duality. We employ only the classical Hardy space theory and an elementary formula expressing the inner product on $\mathcal{H}_{\alpha}$ in terms of a weighted superposition of backward shifts.
\end{abstract}

\section{INTRODUCTION}

Forward shift operators (multiplication by the independent variable) on weighted Bergman spaces are currently the focus of intense research (see for instance the recent texts [11 14]). It is not hard to see that their adjoints are unitarily equivalent to the backward shift operator on spaces which are norm equivalent to certain Dirichlet-type spaces. To study the backward shift operator on a Hilbert space of analytic functions, one usually first considers the forward shift on the corresponding Cauchy dual. In this note we take a different point of view, approaching the backward shift operator on Dirichlet-type spaces using only the classical theory of Hardy spaces. We demonstrate that many results can be obtained completely independently of the forward shift on the corresponding weighted Bergman spaces. This approach allows us to work in a setting (the Hardy space $H^{2}$ [10]) which is classical and well-understood. Consequently, we have at our disposal a wide array of well-known tools, including inner-outer factorization.

Before proceeding, we require some preliminaries. For each $\alpha \geq 0$ let $\mathcal{H}_{\alpha}$ denote the Hilbert space of analytic functions on the unit disk $\mathbb{D}$ with inner product

$$
\langle f, g\rangle_{\alpha}=\sum_{n=0}^{\infty} \frac{\Gamma(\alpha+1+n)}{\Gamma(\alpha+1) n !} a_{n} \overline{b_{n}}
$$

where $f(z)=\sum_{n=0}^{\infty} a_{n} z^{n}$ and $g(z)=\sum_{n=0}^{\infty} b_{n} z^{n}$. Here $\Gamma$ denotes the standard $\Gamma$-function from complex analysis (see [2]). For each $\alpha>0$ we define $\mathcal{H}_{-\alpha}$ to be the natural Cauchy dual to $\mathcal{H}_{\alpha}$. In other words, the Hilbert spaces $\mathcal{H}_{-\alpha}$ carry the

Received by the editors May 8, 2004 and, in revised form, May 31, 2004

2000 Mathematics Subject Classification. Primary 30D55, 47B38.

Key words and phrases. Backward shift operator, Dirichlet-type spaces, weighted Bergman spaces, cyclic function, noncyclic function, invariant subspaces, pseudocontinuation, Bergman shift operator.

(C)2005 American Mathematical Society Reverts to public domain 28 years from publication 
inner product

$$
\langle f, g\rangle_{-\alpha}=\sum_{n=0}^{\infty} \frac{\Gamma(\alpha+1) n !}{\Gamma(\alpha+1+n)} a_{n} \overline{b_{n}}
$$

and we have the dual pairing

$$
\langle f, g\rangle=\sum_{n=0}^{\infty} a_{n} \overline{b_{n}}
$$

(where $f \in \mathcal{H}_{\alpha}, g \in \mathcal{H}_{-\alpha}$ ) between $\mathcal{H}_{\alpha}$ and $\mathcal{H}_{-\alpha}$.

The $\mathcal{H}_{\alpha}$ spaces encompass many standard spaces. For instance,

$$
\begin{aligned}
\mathcal{H}_{0} & =H^{2} & & \text { the Hardy space, } \\
\mathcal{H}_{1} & =\mathcal{D} & & \text { the Dirichlet space, } \\
\mathcal{H}_{-1} & =A^{2} & & \text { the Bergman space, } \\
\mathcal{H}_{-\alpha} & =A_{\alpha-1}^{2}, \alpha>0 & & \text { weighted Bergman spaces. }
\end{aligned}
$$

By Stirling's Formula, we see that $\mathcal{H}_{\alpha} \subseteq \mathcal{H}_{\beta}$ if and only if $\beta \leq \alpha$. Moreover, the $\mathcal{H}_{\alpha}$ spaces are norm equivalent to the Dirichlet-type spaces $D_{\alpha}$ [17] which correspond to the weight sequences $(n+1)^{\alpha}$.

The backward shift of an analytic function $f$ on $\mathbb{D}$ is given by

$$
B f(z)=\frac{f(z)-f(0)}{z} .
$$

On each $\mathcal{H}_{\alpha}$ space the mapping $f \mapsto B f$ is a bounded linear operator (also denoted by $B$ ) and sends the coefficient sequence $\left(a_{0}, a_{1}, a_{2}, \ldots\right)$ to $\left(a_{1}, a_{2}, a_{3}, \ldots\right)$. Observe also that the backward shift on $\mathcal{H}_{\alpha}(\alpha \geq 0)$ is unitarily equivalent to the adjoint of the forward shift $f(z) \mapsto z f(z)$ on the weighted Bergman space $A_{\alpha-1}^{2}$.

The author would like to thank A. Aleman and D. Sarason for their comments and suggestions. Several questions in Section 4 were posed by Sarason and the author is most indebted to him. Furthermore, the comments of the anonymous referee were most helpful and much appreciated.

\section{INNER PRODUCT FORMULA}

The following elementary formula expresses the inner product of two functions $f, g$ in $\mathcal{H}_{\alpha}$ in terms of a weighted sum of the inner products of their backward shifts in $\mathcal{H}_{\beta}$ (where $\alpha, \beta \geq 0$ ). Recalling that the $\Gamma$-function has a simple pole at $-n(n=0,1,2, \ldots)$ with residue $(-1)^{n} / n$ !, we see that $\frac{\Gamma(\alpha+n)}{\Gamma(\alpha) n !}$ makes sense for negative integral $\alpha$ when interpreted in the obvious way. Similar formulas (in terms of integrals) are utilized in [3, 4, 6].

Lemma 1. If $f, g \in \mathcal{H}_{\alpha} \cap \mathcal{H}_{\beta}=\mathcal{H}_{\max \{\alpha, \beta\}}$ for some $\alpha, \beta \geq 0$, then

$$
\langle f, g\rangle_{\alpha}=\sum_{n=0}^{\infty} \frac{\Gamma(\alpha-\beta+n)}{\Gamma(\alpha-\beta) n !}\left\langle B^{n} f, B^{n} g\right\rangle_{\beta} .
$$

Proof. By polarization, it suffices to consider the special case $f=g$. Let $f(z)=$ $\sum_{n=0}^{\infty} a_{n} z^{n}$ and first consider the case $\alpha \geq \beta$. We require the formula

$$
\frac{\Gamma(\alpha+1+m)}{\Gamma(\alpha+1) m !}=\sum_{n=0}^{m} \frac{\Gamma(\beta+1+(m-n))}{\Gamma(\beta+1)(m-n) !} \cdot \frac{\Gamma(\alpha-\beta+n)}{\Gamma(\alpha-\beta) n !}
$$


which follows by comparing Taylor coefficients in the identity

$$
\frac{1}{(1-z)^{\alpha+1}}=\frac{1}{(1-z)^{\beta+1}} \cdot \frac{1}{(1-z)^{\alpha-\beta}} \text {. }
$$

By rearranging a convergent series of nonnegative terms we obtain

$$
\begin{aligned}
\|f\|_{\alpha}^{2} & =\sum_{m=0}^{\infty} \frac{\Gamma(\alpha+1+m)}{\Gamma(\alpha+1) m !}\left|a_{m}\right|^{2} \\
& =\sum_{m=0}^{\infty}\left|a_{m}\right|^{2} \sum_{n=0}^{m} \frac{\Gamma(\beta+1+(m-n))}{\Gamma(\beta+1)(m-n) !} \cdot \frac{\Gamma(\alpha-\beta+n)}{\Gamma(\alpha-\beta) n !} \\
& =\sum_{n=0}^{\infty} \frac{\Gamma(\alpha-\beta+n)}{\Gamma(\alpha-\beta) n !} \sum_{m \geq n} \frac{\Gamma(\beta+1+(m-n))}{\Gamma(\beta+1)(m-n) !}\left|a_{m}\right|^{2} \\
& =\sum_{n=0}^{\infty} \frac{\Gamma(\alpha-\beta+n)}{\Gamma(\alpha-\beta) n !} \sum_{k=0}^{\infty} \frac{\Gamma(\beta+1+k)}{\Gamma(\beta+1) k !}\left|a_{n+k}\right|^{2} \\
& =\sum_{n=0}^{\infty} \frac{\Gamma(\alpha-\beta+n)}{\Gamma(\alpha-\beta) n !}\left\|B^{n} f\right\|_{\beta}^{2}
\end{aligned}
$$

as claimed. If $\beta \geq \alpha$, then the steps may be reversed since all but finitely many of the terms $\frac{\Gamma(\alpha-\beta+n)}{\Gamma(\alpha-\beta) n !}$ share the same sign. This follows from the fact that $\Gamma(\alpha-\beta+n)$ is positive for sufficiently large $n$.

This formula generalizes several well-known identities. For example, setting $\alpha=1$ and $\beta=0$ yields a relationship between the norms on the Dirichlet space $\mathcal{D}$ and the Hardy space $H^{2}$ :

$$
\|f\|_{\mathcal{D}}^{2}=\sum_{n=0}^{\infty}\left\|B^{n} f\right\|_{H^{2}}^{2} .
$$

The two-isometric identity

$$
\left\|z^{2} f\right\|_{\mathcal{D}}^{2}-2\|z f\|_{\mathcal{D}}^{2}+\|f\|_{\mathcal{D}}^{2}=0
$$

for the Dirichlet shift [16] arises from the values $\beta=0$ and $\alpha=1$. Finally, observe that if $f$ does not belong to $\mathcal{H}_{\alpha}$, then both sides of our formula are infinite and equality trivially holds.

Recall that a subspace (a norm-closed linear manifold) $\mathcal{M} \subseteq \mathcal{H}_{\alpha}$ is called $B$ invariant (or simply invariant) if $B \mathcal{M} \subseteq \mathcal{M}$. For each $f$ in $\mathcal{H}_{\alpha}$, let $[f]_{\alpha}$ denote the cyclic subspace of $\mathcal{H}_{\alpha}$ generated by $f$. In other words, $[f]_{\alpha}$ is the norm-closed linear span of the functions $f, B f, B^{2} f, \ldots$ in $\mathcal{H}_{\alpha}$. We say that two subspaces $\mathcal{M}_{1}, \mathcal{M}_{2} \subseteq$ $\mathcal{H}_{\alpha}$ are orthogonal (denoted $\mathcal{M}_{1} \perp_{\alpha} \mathcal{M}_{2}$ ) if $\langle f, g\rangle_{\alpha}=0$ for every $f \in \mathcal{M}_{1}$ and $g \in \mathcal{M}_{2}$.

We prove now that nontrivial invariant subspaces of $\mathcal{H}_{\alpha}$ are never orthogonal. While this is not necessarily surprising, it is nevertheless not obvious for we do not have a complete description of what $B$-invariant subspaces of $\mathcal{H}_{\alpha}$ look like. We require the following lemma.

Lemma 2. If $\mathcal{M}_{1}$ and $\mathcal{M}_{2}$ are B-invariant subspaces of $H^{2}$ such that $\mathcal{M}_{1} \perp \mathcal{M}_{2}$, then either $\mathcal{M}_{1}$ or $\mathcal{M}_{2}$ is trivial. 
Proof. Suppose that $\mathcal{M}_{1}$ and $\mathcal{M}_{2}$ are orthogonal $B$-invariant subspaces of $H^{2}$. Without loss of generality, assume that $\mathcal{M}_{1}$ is nontrivial and let $f$ be a function in $\mathcal{M}_{1}$ that does not vanish identically. If $g \in \mathcal{M}_{2}$, then we have $\left\langle B^{j} f, B^{k} g\right\rangle_{H^{2}}=0$ for every $j, k \in \mathbb{N}$. We may write this as $\left\langle U^{k}\left(U^{*}\right)^{j} f, g\right\rangle_{H^{2}}=0$ where $U$ denotes the unilateral shift operator and $U^{*}=B$ is its adjoint (acting on $H^{2}$ ). The closed linear span $\mathcal{M}$ of the set $\left\{U^{k}\left(U^{*}\right)^{j} f: j, k \in \mathbb{N}\right\}$ is a nontrivial subspace of $H^{2}$ that is orthogonal to $g$ and invariant for both $U$ and $U^{*}$. If $\mathcal{M}$ is not all of $H^{2}$, then by Beurling's Theorem [10, there exist nonconstant inner functions $\varphi_{1}$ and $\varphi_{2}$ such that $\mathcal{M}=\varphi_{1} H^{2}=\left(\varphi_{2} H^{2}\right)^{\perp}$. This implies that $\left\langle\varphi_{1} h_{1}, \varphi_{2} h_{2}\right\rangle=0$ for all $h_{1}, h_{2} \in H^{2}$. Selecting $h_{1}=\varphi_{2}$ and $h_{2}=\varphi_{1}$ we find that $\left\|\varphi_{1} \varphi_{2}\right\|=0$, a contradiction. Therefore $\mathcal{M}=H^{2}$ and $g$ vanishes identically. This implies that the subspace $\mathcal{M}_{2}$ is trivial.

Theorem 1. Let $\alpha \geq 0$. If $\mathcal{M}_{1}$ and $\mathcal{M}_{2}$ are $B$-invariant subspaces of $\mathcal{H}_{\alpha}$ such that $\mathcal{M}_{1} \perp_{\alpha} \mathcal{M}_{2}$, then either $\mathcal{M}_{1}$ or $\mathcal{M}_{2}$ is trivial.

Proof. Select $f \in \mathcal{M}_{1}$ and $g \in \mathcal{M}_{2}$ that do not vanish identically and note that $f, g \in H^{2}$ since $\mathcal{H}_{\alpha} \subseteq H^{2}$ for $\alpha \geq 0$. Since $[f]_{\alpha} \perp_{\alpha}[g]_{\alpha}$, Lemma 1 (with $\alpha=0$, $\beta=\alpha$ ) implies that

$$
\left\langle B^{j} f, B^{k} g\right\rangle_{H^{2}}=\sum_{n=0}^{\infty} \frac{\Gamma(-\alpha+n)}{\Gamma(-\alpha) n !}\left\langle B^{n+j} f, B^{n+k} g\right\rangle_{\alpha}=0
$$

for all $j, k \geq 0$. Hence $[f]_{H^{2}}$ and $[g]_{H^{2}}$ are orthogonal in $H^{2}$, a contradiction.

Corollary. Let $\alpha \geq 0$. If $\mathcal{M}_{1}$ and $\mathcal{M}_{2}$ are two nontrivial invariant subspaces of $\mathcal{H}_{\alpha}$, then the orthogonal projection $P: \mathcal{M}_{1} \rightarrow \mathcal{M}_{2}$ is nonzero.

The preceding proposition and corollary indicate our general approach. Known results about the backward shift on $H^{2}$ can be imported into the $\mathcal{H}_{\alpha}$ setting by using Lemma 1 .

\section{CyClic subspaces}

Recall that a function $f$ in $\mathcal{H}_{\alpha}$ is called cyclic (in $\mathcal{H}_{\alpha}$ ) if $[f]_{\alpha}=\mathcal{H}_{\alpha}$ and noncyclic otherwise. Our primary tool for studying cyclic subspaces are conjugate-analytic Toeplitz operators, which we show operate boundedly on the spaces $\mathcal{H}_{\alpha}$. Although this can be proved using the Sz.-Nagy-Foiaş functional calculus for pure contractions [19], we use Lemma 1 to obtain a direct proof which is independent of the Cauchy duality.

Recall that if $u \in L^{\infty}(\partial \mathbb{D})$, then the Toeplitz operator with symbol $u$ is the operator $T_{u}: H^{2} \rightarrow H^{2}$ defined by $T_{u} f=P(u f)$ where $P$ denotes the orthogonal projection from $L^{2}$ onto $H^{2}$. A Toeplitz operator is called conjugate analytic if it is of the form $T_{\bar{g}}$ for some $g \in H^{\infty}$. Such an operator commutes with $B$ and can be represented as $\widetilde{g}(B)$ where $\widetilde{g}(z)=\overline{g(\bar{z})}$ is in $H^{\infty}$. If $T$ is a bounded linear operator on $\mathcal{H}_{\alpha}$, then we denote the operator norm of $T$ acting on $\mathcal{H}_{\alpha}$ by $\|T\|_{\alpha}$.

Proposition 1. If $\alpha \geq 0$, then $\|g(B) f\|_{\alpha} \leq\|g\|_{\infty}\|f\|_{\alpha}$ for every $f \in \mathcal{H}_{\alpha}$ and $g \in H^{\infty}$. Moreover, $\|g(B)\|_{\alpha}=\|g\|_{\infty}$. 
Proof. If $\alpha \geq 0$ and $f \in \mathcal{H}_{\alpha}$, then Lemma 1 implies that

$$
\begin{aligned}
\|g(B) f\|_{\alpha}^{2} & =\sum_{n=0}^{\infty} \frac{\Gamma(\alpha+n)}{\Gamma(\alpha) n !}\left\|B^{n} g(B) f\right\|_{H^{2}}^{2} \\
& =\sum_{n=0}^{\infty} \frac{\Gamma(\alpha+n)}{\Gamma(\alpha) n !}\left\|g(B)\left(B^{n} f\right)\right\|_{H^{2}}^{2} \\
& \leq\|g(B)\|_{H^{2}}^{2} \sum_{n=0}^{\infty} \frac{\Gamma(\alpha+n)}{\Gamma(\alpha) n !}\left\|B^{n} f\right\|_{H^{2}}^{2} \\
& =\|g\|_{\infty}^{2}\|f\|_{\alpha}^{2}
\end{aligned}
$$

and thus $\|g(B)\|_{\alpha} \leq\|g\|_{\infty}$. Note that the last equality follows from the fact that the operator norm of a Toeplitz operator on $H^{2}$ equals the $\infty$-norm of its symbol [13. p. 138]. Since $g(B) e_{\lambda}=g(\lambda) e_{\lambda}$ where $e_{\lambda}(z)=(1-\lambda z)^{-1}$ and $\lambda \in \mathbb{D}$, the reverse inequality $\|g\|_{\infty} \leq\|g(B)\|_{\alpha}$ follows.

Fix $\alpha \geq 0$ and $f \in \mathcal{H}_{\alpha}$. We now prove that $g(B) f$ belongs to the cyclic invariant subspace $[f]_{\alpha}$ for each $g \in H^{\infty}$. This amounts to showing that there exist polynomials $g_{n}$ such that $g_{n}(B)$ converges to $g(B)$ in the strong operator topology (for operators on $\mathcal{H}_{\alpha}$ ). As usual, we proceed directly and make no use of duality.

Proposition 2. If $g \in H^{\infty}, \alpha \geq 0$, and $f \in \mathcal{H}_{\alpha}$, then $g(B) f \in[f]_{\alpha}$.

Proof. Let $g \in H^{\infty}$ and let $g_{n}$ denote the sequence of Fejér polynomials for $g$. This sequence has the property that $g_{n} \rightarrow g$ locally uniformly on $\mathbb{D}$ and $\left\|g_{n}\right\|_{\infty} \leq\|g\|_{\infty}$ for all $n$. Given $f \in \mathcal{H}_{\alpha}$ and $\epsilon>0$, we can find a polynomial $p$ such that

$$
\|f-p\|_{\alpha}<\frac{\epsilon}{2\|g\|_{\infty}}
$$

Proposition 1 implies that

$$
\begin{aligned}
\left\|g(B) f-g_{n}(B) f\right\|_{\alpha} & =\left\|\left(g-g_{n}\right)(B) f\right\|_{\alpha} \\
& =\left\|\left(g-g_{n}\right)(B)(f-p+p)\right\|_{\alpha} \\
& \leq\left\|\left(g-g_{n}\right)(B)(f-p)\right\|_{\alpha}+\left\|\left(g-g_{n}\right)(B) p\right\|_{\alpha} \\
& \leq\left\|g-g_{n}\right\|_{\infty}\|f-p\|_{\alpha}+\left\|\left(g-g_{n}\right)(B) p\right\|_{\alpha} \\
& \leq\left(\|g\|_{\infty}+\left\|g_{n}\right\|_{\infty}\right)\|f-p\|_{\alpha}+\left\|\left(g-g_{n}\right)(B) p\right\|_{\alpha} \\
& \leq 2\|g\|_{\infty}\|f-p\|_{\alpha}+\left\|\left(g-g_{n}\right)(B) p\right\|_{\alpha} \\
& \leq \epsilon+\left\|\left(g-g_{n}\right)(B) p\right\|_{\alpha} .
\end{aligned}
$$

To show that $\left\|g(B) f-g_{n}(B) f\right\|_{\alpha} \rightarrow 0$, it suffices to demonstrate that

$$
\lim _{n \rightarrow \infty}\left\|\left(g-g_{n}\right)(B) z^{N}\right\|_{\alpha}=0
$$


for each monomial $z^{N}$. Letting $\left[g-g_{n}\right]_{k}$ denote the $k$ th Taylor coefficient at the origin for the function $g-g_{n}$ we see that

$$
\begin{aligned}
\left\|\left(g-g_{n}\right)(B) z^{N}\right\|_{\alpha} & =\left\|\sum_{k=0}^{\infty}\left[g-g_{n}\right]_{k} B^{k} z^{N}\right\|_{\alpha} \\
& =\left\|\sum_{k=0}^{N}\left[g-g_{n}\right]_{k} B^{k} z^{N}\right\|_{\alpha} \\
& =\left\|\sum_{k=0}^{N}\left[g-g_{n}\right]_{k} z^{(N-k)}\right\|_{\alpha} \\
& \leq\left\|z^{N}\right\|_{\alpha}(N+1) \max _{0 \leq k \leq N}\left|\left[g-g_{n}\right]_{k}\right| .
\end{aligned}
$$

Since $g_{n} \rightarrow g$ locally uniformly, this tends to 0 and concludes the proof.

We say that a linear submanifold $\mathcal{M}$ of $H^{2}$ has the $F$-property if $f / I$ belongs to $\mathcal{M}$ whenever $f \in \mathcal{M}$ and $I$ is an inner function dividing the inner factor of $f$. For example, the $B$-invariant subspaces of $H^{2}$ enjoy the $F$-property [9] Thm. 3.1.13] as do the Dirichlet-type spaces $\mathcal{H}_{\alpha}$ for $\alpha \geq 0$ [18].

Theorem 2. Let $\alpha \geq 0$ and $f \in \mathcal{H}_{\alpha}$. If $I$ is an inner function that divides the inner factor of $f$, then $f / I \in[f]_{\alpha}$.

Proof. Take $g=\widetilde{I}$ in Proposition 2 to obtain the desired conclusion.

Since a cyclic invariant subspace $[f]_{\alpha}$ of $\mathcal{H}_{\alpha}$ is spanned by functions of the form $p(B) f$ where $p$ is a polynomial, it is natural to ask whether the linear manifold

$$
H^{\infty}(B) f:=\left\{g(B) f: g \in H^{\infty}\right\}
$$

actually equals $[f]_{\alpha}$. We handle this question using a compactness argument.

Theorem 3. $H^{\infty}(B) f=[f]_{\alpha}$ if and only if $f$ is a rational function.

Proof. For each fixed $\alpha \geq 0$ and function $f \in \mathcal{H}_{\alpha}$, Proposition 1 implies that the (Banach space) operator $g \mapsto g(B) f$ from $H^{\infty}$ to $\mathcal{H}_{\alpha}$ is bounded by $\|f\|_{\alpha}$. If $f_{n}$ denotes the $n$th Taylor polynomial of $f$, then

$$
\left\|g(B) f-g(B) f_{n}\right\|_{\alpha} \leq\|g\|_{\infty}\left\|f-f_{n}\right\|_{\alpha} \rightarrow 0
$$

and hence $g \mapsto g(B) f$ is compact, being the limit of the finite rank operators $g \mapsto g(B) f_{n}$. As a compact operator, $g \mapsto g(B) f$ can have closed range if and only if that range is finite dimensional. Therefore $H^{\infty}(B) f=[f]_{\alpha}$ if and only if $[f]_{\alpha}$ is finite dimensional, which happens if and only if $f$ is a rational function.

In other words, if $[f]_{\alpha}$ is infinite dimensional (for instance, if $f$ is cyclic), then $H^{\infty}(B) f$ fails to "fill up" $[f]_{\alpha}$. Under some circumstances, these ideas can be generalized. Observe that if $f(z)=\sum_{n=0}^{\infty} a_{n} z^{n} \in \mathcal{H}_{\alpha}$ and $g(z)=\sum_{n=0}^{\infty} b_{n} z^{n} \in$ $\mathcal{H}_{-\alpha}$, then

$$
\begin{aligned}
g(B) f(z) & =\sum_{n=0}^{\infty} b_{n} B^{n} f(z) \\
& =\sum_{n=0}^{\infty}\left\langle B^{n} f, \widetilde{g}\right\rangle z^{n}
\end{aligned}
$$


defines an analytic function on $\mathbb{D}$. If restrictions are placed on $f$, then we can say significantly more.

Proposition 3. If $\alpha, \beta \geq 0, g \in \mathcal{H}_{-\beta}$, and $f \in \mathcal{H}_{\alpha+\beta+1}$, then

$$
\|g(B) f\|_{\alpha} \leq\|g\|_{-\beta}\|f\|_{\alpha+\beta+1}
$$

and $g(B) f \in[f]_{\alpha}$.

Proof. The duality of $\mathcal{H}_{\beta}$ and $\mathcal{H}_{-\beta}$ implies that $g(B) f$ is well defined. The preceding discussion and Lemma 1 yield

$$
\begin{aligned}
\|g(B) f\|_{\alpha}^{2} & =\sum_{n=0}^{\infty} \frac{\Gamma(\alpha+1+n)}{\Gamma(\alpha+1) n !}\left|\left\langle B^{n} f, \widetilde{g}\right\rangle\right|^{2} \\
& \leq\|\widetilde{g}\|_{-\beta}^{2} \sum_{n=0}^{\infty} \frac{\Gamma(\alpha+1+n)}{\Gamma(\alpha+1) n !}\left\|B^{n} f\right\|_{\beta}^{2} \\
& =\|g\|_{-\beta}^{2}\|f\|_{\alpha+\beta+1}^{2}
\end{aligned}
$$

which is the desired inequality. Approximating $g$ in $\mathcal{H}_{-\beta}$ by its Taylor polynomials shows that $g(B) f \in[f]_{\alpha}$.

For $\alpha, \beta \geq 0$ we see that $g(B)$ (formally a conjugate-analytic Toeplitz operator) maps the smaller space $\mathcal{H}_{\alpha+\beta+1}$ boundedly into the larger space $\mathcal{H}_{\alpha}$ whenever the "symbol" $g$ belongs to $\mathcal{H}_{-\beta}$. This is of interest since the spaces $\mathcal{H}_{-\beta}$ are much larger than $H^{\infty}$.

Example. For any $g$ in $H^{2}=\mathcal{H}_{0}, g(B)$ maps $\mathcal{D}=\mathcal{H}_{1}$ boundedly into $H^{2}$. Fixing an $f \in \mathcal{D}$, Proposition 3 (with $\alpha=\beta=0$ ) implies that the function $g(B) f$ belongs to $[f]_{H^{2}}$ for any $g$ in $H^{2}$. In other words, since the Taylor coefficients of a Dirichlet function tend to zero faster than those of a typical $H^{2}$ function, $g(B) f$ belongs to $H^{2}$ for a much wider range of "symbols" $g$ than one might expect.

If $f \in \mathcal{H}_{\alpha+\beta+1}$ for some $\alpha, \beta \geq 0$, then a proof similar to that of Theorem 3 shows that the operator $g \mapsto g(B) f$ from $\mathcal{H}_{-\beta}$ to $\mathcal{H}_{\alpha}$ is compact and that $\mathcal{H}_{-\beta}(B) f:=$ $\left\{g(B) f: g \in \mathcal{H}_{-\beta}\right\}$ is properly contained in $[f]_{\alpha}$ unless $f$ is a rational function. This yields the following theorem:

Theorem 4. If $f \in \mathcal{H}_{\alpha+\beta+1}$ for some $\alpha, \beta \geq 0$, then $\mathcal{H}_{-\beta}(B) f=[f]_{\alpha}$ if and only if $f$ is a rational function.

Example. The function $f(z)=(1-z)^{\frac{1}{2}}$ belongs to the Dirichlet space $\mathcal{H}_{1}=\mathcal{D}$ and is cyclic in $\mathcal{H}_{0}=H^{2}$ by [9] Thm. 2.2.3] since it has an isolated winding singularity at the point $z=1$. Despite the fact that functions of the form $p(B) f$ where $p$ is a polynomial are dense in $H^{2}$, the linear manifold $H^{2}(B) f$ is not all of $H^{2}$. In other words, even by enlarging the space of "symbols" from the usual $H^{\infty}$ to the entire Hardy space $H^{2}$, we do not obtain all of $[f]_{H^{2}}$. Consequently, there are functions in $H^{2}$ that cannot be expressed in the form $g(B) f$ where $g$ belongs to $H^{2}$.

\section{INVARIANT SUBSPACES BY INTERSECTION}

One method of constructing $B$-invariant subspaces of $\mathcal{H}_{\alpha}$ (for $\alpha>0$ ) is to simply take an invariant subspace of $H^{2}=\mathcal{H}_{0}$ and intersect it with $\mathcal{H}_{\alpha}$. In light of Beurling's Theorem, we are concerned primarily with invariant subspaces of the form $\mathcal{M}_{\varphi} \cap \mathcal{H}_{\alpha}$ where $\varphi$ is a nonconstant inner function and $\mathcal{M}_{\varphi}:=H^{2} \ominus \varphi H^{2}$, the 
orthogonal complement of the subspace $\varphi H^{2}$ in $H^{2}$. Although easy in principle, the construction has its subtleties. We require the following easy lemma, whose proof we omit.

Lemma 3. If $\mathcal{M}$ is a proper $B$-invariant subspace of $\mathcal{H}_{\alpha}$ for some $\alpha \geq 0$, then $\mathcal{M} \cap \mathcal{H}_{\beta}$ is a proper $B$-invariant subspace of $\mathcal{H}_{\beta}$ for all $\beta \geq \alpha$.

Several questions immediately come to mind. First, are all invariant subspaces of $\mathcal{H}_{\alpha}$ of the form $\mathcal{M}_{\varphi} \cap \mathcal{H}_{\alpha}$ ? Second, is $\mathcal{M}_{\varphi} \cap \mathcal{H}_{\alpha}$ always nontrivial? We provide partial answers to these questions and point out a major structural difference between invariant subspaces of $H^{2}=\mathcal{H}_{0}$ and $\mathcal{H}_{\alpha}$ for $\alpha>0$.

The answer to the first question is negative, at least for $\mathcal{H}_{1}=\mathcal{D}$ (the Dirichlet space). Aleman, Richter, and Ross [5, Thm. 6.2] proved that there exist noncyclic functions in the Dirichlet space which are not pseudocontinuable of bounded type (see [17] for details). Such functions cannot belong to an invariant subspace of the form $\mathcal{M}_{\varphi} \cap \mathcal{H}_{\alpha}$ by a well-known theorem of Douglas, Shapiro, and Shields [9]. Hence not every invariant subspace of the Dirichlet space is of the form $\mathcal{M}_{\varphi} \cap \mathcal{D}$.

A variety of results on the Taylor coefficients of inner functions exist in the literature (see [1], for instance) which provide examples of nontrivial invariant subspaces of the form $\mathcal{M}_{\varphi} \cap \mathcal{H}_{\alpha}$.

Example. Newman and Shapiro [15] showed that if $\varphi(z)=\sum_{n=0}^{\infty} a_{n} z^{n}$ is a Blaschke product whose zeroes $z_{n}$ satisfy $1-\left|z_{n+1}\right| \leq \delta\left(1-\left|z_{n}\right|\right)$ for some $\delta \in(0,1)$, then $a_{n}=O(1 / n)$. If $\varphi$ is such a Blaschke product, then $\mathcal{M}_{\varphi} \cap \mathcal{H}_{\alpha}$ is nontrivial for every $\alpha \in[0,1)$.

Example. In [15], Newman and Shapiro also considered the Taylor coefficients of singular inner functions. They showed that a singular inner function may have coefficients that are $O\left(n^{-3 / 4}\right)$. If $\varphi$ is such a singular inner function, then the subspace $\mathcal{M}_{\varphi} \cap \mathcal{H}_{\alpha}$ is nontrivial for every $\alpha \in\left[0, \frac{1}{2}\right)$.

The previous examples relied on the assumption that $\varphi$ belongs to $\mathcal{H}_{\alpha}$ (for some specific value of $\alpha$ ). A refinement of our second question is in order, namely, if $\alpha>0$ and an inner function $\varphi$ does not belong to $\mathcal{H}_{\alpha}$, is $\mathcal{M}_{\varphi} \cap \mathcal{H}_{\alpha}$ nontrivial?

Example. If the inner function $\varphi$ has a nontrivial Blaschke factor, then $\mathcal{M}_{\varphi} \cap \mathcal{H}_{\alpha}$ is nontrivial for every $\alpha \geq 0$. Indeed, the Taylor coefficients of a finite Blaschke product (being a rational function whose poles lie in the region $|z|>1$ ) decay exponentially and hence $\mathcal{M}_{z \varphi} \cap \mathcal{H}_{\alpha}$ contains every finite Blaschke subproduct obtained from the Blaschke factor of $\varphi$. From this it is easy to see that $\mathcal{M}_{\varphi} \cap \mathcal{H}_{\alpha}$ is nontrivial.

Example. The question becomes much more subtle if $\varphi$ is purely singular. If $\varphi$ is a singular inner function whose singular measure $\mu$ has a modulus of continuity $\omega(t ; \mu)$ satisfying $\omega(t ; \mu)=O(t \log 1 / t)$, then $\mathcal{M}_{\varphi} \cap \mathcal{H}_{\alpha}$ is trivial for every $\alpha>0$ by [10, Thm. 7.6].

The structure of a (nontrivial) invariant subspace of the form $\mathcal{M}_{\varphi} \cap \mathcal{H}_{\alpha}$ can be far different than that of $\mathcal{M}_{\varphi}$ itself. In [12], it was shown that for every outer function $F$ that appears as the outer factor of a function in $\mathcal{M}_{\varphi}$, there exists a unique (up to a unimodular constant factor) inner function $\mathcal{I}_{F}$ such that an inner function $I_{f}$ divides $\mathcal{I}_{F}$ if and only if $f=I_{f} F$ lies in $\mathcal{M}_{\varphi}$. In other words, the functions in $\mathcal{M}_{\varphi}$ with outer factor $F$ are partially ordered by the divisibility of their respective inner factors and this partial ordering has a maximal element, namely $\mathcal{I}_{F} F$. Certain 
invariant subspaces of $\mathcal{H}_{\alpha}$ for $\alpha \geq 1$ do not enjoy this structural property. The problem is that $f=I_{f} F$ may belong to $\mathcal{M}_{\varphi} \cap \mathcal{H}_{\alpha}$ even though $\mathcal{I}_{F} F$ does not.

Example. Consider the outer function $F=1$ and let $\varphi$ be an infinite Blaschke product. By Carleson's formula for the Dirichlet integral [7, we see that $\varphi$ does not belong to $\mathcal{H}_{\alpha}$ for any $\alpha \geq 1$. Nevertheless, every finite subproduct of $\varphi$ belongs to every $\mathcal{H}_{\alpha}$ space. Therefore the partially ordered collection of functions in $\mathcal{M}_{z \varphi} \cap \mathcal{H}_{\alpha}$ with trivial outer factor contains no maximal element for any $\alpha \geq 1$. With additional effort, it appears likely that similar examples can be found for $\alpha \in(0,1)$.

A related question appears open. Suppose that $\mathcal{M}$ is an invariant subspace of $\mathcal{H}_{\alpha}$ for some $\alpha \geq 0$. We say that $\mathcal{M}$ has the least common multiple property if for every pair $I_{1} F, I_{2} F \in \mathcal{M}$ with common outer factor $F$ and inner factors $I_{1}, I_{2}$, the function $I F$ belongs to $\mathcal{M}$ where $I$ denotes the least common multiple of $I_{1}$ and $I_{2}$. This is certainly true in the case $\mathcal{H}_{0}=H^{2}$ since both $I_{1}$ and $I_{2}$ must divide $\mathcal{I}_{F}$ [12. For $\alpha>0$ we encounter two obstacles. First, there may be invariant subspaces of $\mathcal{H}_{\alpha}$ which are not of the type $\mathcal{M}_{\varphi} \cap \mathcal{H}_{\alpha}$. Our intuition regarding such subspaces is lacking. Second, we do not know whether $I_{1} F, I_{2} F \in \mathcal{M}$ implies that $I F$ belongs to $\mathcal{H}_{\alpha}$, let alone $\mathcal{M}$.

Retaining the notation of the preceding paragraph, we conclude this section by demonstrating that $B$-invariant subspaces of the Dirichlet space $\mathcal{D}=\mathcal{H}_{1}$ which are of the form $\mathcal{M}=\mathcal{M}_{\varphi} \cap \mathcal{D}$ (where $\varphi$ is a nonconstant inner function) possess the least common multiple property. We state without proof the following easy lemma.

Lemma 4. If $F$ is an outer function and $I_{1}, I_{2}$ are inner functions such that $I_{1} F$ and $I_{2} F$ belong to $\mathcal{D}$, then $I_{1} I_{2} F$ belongs to $\mathcal{D}$.

Proposition 4. Invariant subspaces of $\mathcal{D}$ of the form $\mathcal{M}_{\varphi} \cap \mathcal{D}$ have the least common multiple property.

Proof. If $F$ is an outer function such that $I_{1} F$ and $I_{2} F$ belong to an invariant subspace of the form $\mathcal{M}=\mathcal{M}_{\varphi} \cap \mathcal{D}$, then $I_{1} I_{2} F$ belongs to $\mathcal{D}$ by Lemma 4 . Since inner factors of $I_{1} I_{2} F$ can be removed without leaving $\mathcal{D}$ by Theorem 2 we conclude that $I F \in \mathcal{D}$ and thus $\mathcal{M}$ has the least common multiple property.

\section{REFERENCES}

[1] Ahern, P., The mean modulus and derivative of an inner function, Math. J., 28, no.2 (1979), 311-347. Mr 0523107 (80h:30027)

[2] Ahlfors, L.V., Complex Analysis (Third Edition), International Series in Pure and Applied Mathematics, McGraw-Hill, 1979. MR0510197 (80c:30001)

[3] Aleman, A. The multiplication operator on Hilbert spaces of analytic functions, Habilitationsschrift, Hagen, 1993.

[4] Aleman, A., Richter, S., Sundberg, C., Beurling's theorem for the Bergman space, Acta Math., 177 (1996), 275-310. MR.1440934 (98a:46034)

[5] Aleman, A., Richter, S., Ross, W.T., Pseudocontinuations and the backward shift, Indiana Univ. Math. J., 47 (1998), no.1, 223-276. MF,1631561 (2000i:47009)

[6] Aleman, A., Richter, S., Simply invariant subspaces of $\mathrm{H}^{2}$ of some multiply connected regions, Integral Equations Operator Theory, 24 (1996), 127-155. MR1371943 (99b:47010a)

[7] Carleson, L., A representation theorem for the Dirichlet integral, Math. Z. 73 (1960), 190196. MR0112958 (22:3803)

[8] Cima, J.A., Ross, W.T., The Backward Shift on the Hardy Space, American Mathematical Society, 2000. MR1761913 (2002f:47068) 
[9] Douglas, R.G.; Shapiro, H.S.; Shields, A.L., Cyclic vectors and invariant subspaces for the backward shift operator, Ann. Inst. Fourier (Grenoble) 20, no. 1 (1970), 37-76. MR0270196 (42:5088)

[10] Duren, P., Theory of $H^{p}$ Spaces, Pure and Appl. Math., Vol. 38, Academic Press, 1970. MR0268655 (42:3552)

[11] Duren, P., Schuster, A., Bergman Spaces, Mathematical Surveys and Monographs, Vol. 100, American Mathematical Society, 2004. MR,2033762

[12] Garcia, S.R., Conjugation, the backward shift, and Toeplitz kernels, J. Operator Theory, to appear.

[13] Halmos, P.R., A Hilbert Space Problem Book (Second Edition), Graduate Texts in Mathematics 19, Springer-Verlag, 1982. MR.0675952 (84e:47001)

[14] Hedenmalm, H., Korenblum, B., Zhu, K., Theory of Bergman Spaces, Graduate Texts in Mathematics 199, Springer-Verlag, 2000. MR.1758653 (2001c:46043)

[15] Newman, D.J., Shapiro, H.S., The Taylor coefficients of inner functions, Michigan Math. J. 9 (1962), 249-255. MR0148874(26:6371)

[16] Richter, S., A representation theorem for cyclic analytic two-isometries, Trans. Amer. Math. Soc. 328, no.1 (1991), 325-349. MR1013337 (92e:47052)

[17] Ross, W.T., Shapiro, H.S., Generalized Analytic Continuation, University Lecture Series, Volume 25, American Mathematical Society, 2002. MR1895624 (2003h:30003)

[18] Shirokov, N.A., Analytic Functions Smooth Up to the Boundary, Lecture Notes in Mathematics 1312, Springer-Verlag, 1988. MR0947146 (90h:30087)

[19] Sz.-NaGy, B., Folaş, C., Harmonic Analysis of Operators on Hilbert space, North-Holland, Amsterdam-London, 1970. MR0275190 (43:947)

Department of Mathematics, University of California at Santa Barbara, Santa BarBara, California, 93106-3080

E-mail address: garcias@math.ucsb.edu

$U R L:$ http://math.ucsb.edu/〜garcias 